\title{
A High-Quality Genome Resource of Botrytis fragariae, a New and Rapidly Spreading Fungal Pathogen Causing Strawberry Gray Mold in the United States
}

\author{
Ying Wu, ${ }^{1,2}$ Christopher Saski, ${ }^{3}$ Guido Schnabel, ${ }^{3}$ Shunyuan Xiao, ${ }^{1,2}$ and Mengjun $\mathrm{Hu}^{1, \dagger}$ \\ ${ }^{1}$ Department of Plant Science and Landscape Architecture, University of Maryland, College Park, MD \\ 20742 \\ ${ }^{2}$ Institute for Bioscience and Biotechnology Research, University of Maryland, Rockville, MD 20850 \\ ${ }^{3}$ Department of Plant and Environmental Sciences, Clemson University, Clemson, SC 29634
}

\begin{abstract}
Although Botrytis fragariae was only recently identified as a new Botrytis species that causes strawberry gray mold, its prevalence extends to many states of the eastern United States. Compared with $B$. cinerea, which is known to be the causal agent of gray mold on many crop plants including strawberry, $B$. fragariae appears to have specifically adapted to strawberry and exhibits distinct fungicide sensitivity. This is the first presentation of a high-quality genome assembly of $B$. fragariae with gene annotation based on sequence homology and deep transcriptome data. The genome sequence information from $B$. fragariae is expected to help reveal genomic features underlying its host specialization and evolution of distinct fungicide resistance and other novel pathogenicity mechanisms.
\end{abstract}

\section{Genome Announcement}

Gray mold is arguably the most important fungal disease in many strawberry growing regions worldwide (Petrasch et al. 2019). As the second largest strawberry producer in the world (FAOSTAT 2018), the United States produced 1.3 billion kilograms of strawberries in 2018 , valued at $\$ 2.7$ billion (USDA-NASS 2018). The main causal agent of gray mold, Botrytis cinerea, was ranked as the second most important fungal pathogen by the plant pathology community (Dean et al. 2012) and was previously thought to be the sole causal agent of strawberry gray mold in the United States (Sutton 1998). However, a new species, Botrytis fragariae, has recently been identified to also cause gray mold on strawberry in the United States and Germany (Rupp et al. 2017). A recent study has shown that $35.1 \%$ of the 188 Botrytis isolates collected in five eastern U.S. states were B. fragariae (Dowling et al. 2018), indicating that $B$. fragariae is widely spread in strawberry fields in this region.

Despite the wide distribution of $B$. fragariae, only a few studies have been conducted to characterize its biological features at the phenotypic level. Compared with $B$. cinerea, $B$. fragariae was slightly more aggressive on strawberry flowers albeit causing smaller lesions on leaves and fruits (Rupp et al. 2017). Interestingly, B. fragariae was unable to infect Gerbera petals, apple fruit, or tomato leaves, all of which can be readily infected by $B$. cinerea, suggesting that $B$. fragariae has a narrower host range and may have specifically adapted to strawberries (Rupp et al. 2017). There also are marked differences regarding fungicide sensitivity between the two Botrytis species. Single-site fungicides, including phenylpyrroles (PPs) and succinate dehydrogenase inhibitors (SDHIs), are key chemical classes for strawberry gray mold control. Our recent work showed that some U.S. B. fragariae isolates have higher levels of resistance to

${ }^{\dagger}$ Corresponding author: M. Hu; mjhu@umd.edu

The author(s) declare no conflict of interest.

Accepted for publication 7 July 2020.

\section{Keywords}

disease control and pest management, fungal pathogens, genomics, techniques 
Table 1. A comparison of the Botrytis fragariae genome assembly and gene annotation with those of three other Botrytis species

\begin{tabular}{|c|c|c|c|c|}
\hline Species & B. fragariae & B. paeoniae ${ }^{a}$ & B. tulipae ${ }^{\mathrm{a}}$ & B. cinerea \\
\hline Scaffolds & 28 & 1,833 & 653 & 18 \\
\hline Assembly size (Mb) & 41.92 & 46.36 & 45.45 & 42.65 \\
\hline Largest scaffolds (Mb) & 3.9 & 0.9 & 0.74 & 4.1 \\
\hline BUSCO completeness & $98.50 \%$ & $99.00 \%$ & $98.80 \%$ & $99.80 \%$ \\
\hline Predicted genes & 12,506 & 12,138 & 12,656 & 13,703 \\
\hline $\mathrm{smCOGs}^{\mathrm{c}}$ & 527 & 506 & 526 & 594 \\
\hline Effectorome $^{f}$ & 154 & 145 & 153 & 173 \\
\hline Species specific effector & 67 & 42 & 46 & 57 \\
\hline
\end{tabular}

a The assembly information is from Valero-Jimenez et al. (2019).

b The genome sequence is from NCBI (Botrytis cinerea B05.10, assembly ASM14353V4).

c Secondary metabolite clusters of orthologous groups.

d CAZyme: annotated on dbCAN2 website (http://bcb.unl.edu/dbCAN2/index.php).

e Secretome: proteins have signal peptide, but without a transmembrane domain.

${ }^{f}$ Effectorome: predicted by software EffectorP.

fludioxonil compared with other resistant Botrytis isolates from Europe and the United States (Hu et al. 2019). These isolates overexpressed atrB, a gene that encodes a multidrug efflux pump belonging to the $A B C$ transporter family (Vermeulen et al. 2001). Overexpression of atrB is normally linked to various activating mutations in the transcription factor $m r r 1$ in resistant B. cinerea or German B. fragariae isolates (Kretschmer et al. 2009; Rupp et al. 2017). However, no mutations in $m r r 1$ were found in the U.S. B. fragariae isolates with resistance to fludioxonil, warranting an investigation to reveal a possible novel mechanism (Hu et al. 2019). Furthermore, no resistance to boscalid (SDHI) has been found in B. fragariae, whereas an average of $20 \%$ of $B$. cinerea isolates were found to be resistant to boscalid (Hu et al. 2016). Perhaps resistanceconferring mutations in SDH subunit(s) carry a significant fitness cost in $B$. fragariae that make such isolates unable to compete with the endemic population. A high-quality genome sequence will be useful for understanding the genomic and molecular bases of the unique attributes of $B$. fragariae. Thus, we selected U.S. B. fragariae isolate BVB16 for genome sequencing and assembly, with the expectation that it will serve as the reference genome of $B$. fragariae for exploring the molecular mechanisms underlying its distinct host adaptation, fungicide resistance and other biological attributes.

We sequenced the $B$. fragariae genome using long read Pacific Biosciences technology (Rhoads and Au 2015). High molecular weight genomic DNA ( $50 \mathrm{~kb})$ was extracted using the previously published CTAB-PEG method protocols (Huang et al. 2018; Rhoads and Au 2015). Library preparation and PacBio sequencing were performed at the University of Delaware PacBio Sequencing Facility (https://dna.dbi.udel.edu/). In total, 252M reads were generated using three smart cells, which reached a sequence depth of $\sim 90 \times$ coverage of the $B$. fragariae genome. Genome assembly was conducted using MECAT software (Xiao et al. 2017). The assembled genome size is $41.92 \mathrm{Mb}$, which is comparable to the genome size of other sequenced Botrytis species (43 to $55 \mathrm{Mb}$ ) (Valero-Jimenez et al. 2019). Although the genome assembly for $B$. fragariae has not reached to the level of the gapless, completely assembled 18 chromosomes for the $B$. cinerea genome (Van Kan et al. 2017), the sequenced reads were assembled into only 28 supercontigs with a large N50 length of $2.43 \mathrm{Mb}$ and $98.5 \%$ BUSCO completeness (Table 1), which is more contiguous and complete than the genome assemblies recently reported for other Botrytis sister species, including B. paeoniae and B. tulipae (ValeroJimenez et al. 2019). This indicates that we have obtained a high-quality whole-genome assembly for $B$. fragariae, which ensures the following accurate gene prediction and annotation.

We performed gene prediction using MAKER (2.31.8) (Holt and Yandell 2011) with three iterative runs in order to combine evidence from: (i) three ab initio prediction using SNAP (Korf 2004), AUGUSTUS (Stanke et al. 2004), and GeneMark (Ter-Hovhannisyan et al. 2008); (ii) homology-based comparison against available protein sequences of other Botrytis species from NCBI (https://www.ncbi.nlm.nih.gov/datasets/genomes/?txid=33196) and protein database from Uniprot; and (iii) expression data analysis (i.e., EST assembled from $B$. fragariae mycelial RNA-seq reads generated in this study using Trinity and EST data of $B$. cinerea downloaded from $\mathrm{NCBI}$ ). In the first round we performed complete de novo gene annotation for $B$. fragariae 
using AUGUSTUS with training models from $B$. cinerea and the est2genome and protein2genome were set to 1 based on the annotation protocol. Next, we used the gene models from the first MAKER run to train SNAP while incorporating GeneMark ab initio gene prediction in the second run. Then, we used the gene models from the second MARKER run to train SNAP again to generate the final gene set. Lastly, we performed manual curation with the final gene set using Web Apollo (Lee et al. 2013). In total, we predicted 12,506 genes from the $B$. fragariae genome, which is estimated to cover $98.8 \%$ of all genes in the $B$. fragariae genome based on the proteome-completeness assessment using BUSCO v3 (Simao et al. 2015) against the Ascomycota odb9 database (which contains 1,315 conserved Ascomycota orthologs; https:// busco-archive.ezlab.org/v3/).

With such a high-quality gene prediction, we further performed functional annotation for the deduced B. fragariae proteins using EGGNOG-MAPPER (http://eggnog-mapper.embl.de/) (Huerta-Cepas et al. 2017), which offers insights into the biological characteristics of $B$. fragariae. We found that $8,183 \mathrm{~B}$. fragariae genes (accounting for $65.43 \%$ of the total annotated genes) can be mapped to clusters of ortholog groups (COGs), of which 527 belong to secondary metabolite COGs, suggesting that the majority of $B$. fragariae genes are highly conserved genes. Moreover, we identified 1,184 genes encoding putative secreted proteins using SignalP3.0 (Bendtsen et al. 2004) (which predicts the presence of a signal peptide) and TMHMM 2.0 (Krogh et al. 2001) (which predicts the presence of transmembrane domains) (Table 1). Finally, by using EffectorP, which can predict fungal effector proteins from secretomes (Sperschneider et al. 2018), we predicted 154 putative effector-encoding genes in $B$. fragariae (Table 1).

Compared with $B$. cinerea that has a broad host range (over 1,000 plant species), B. fragariae seems restricted to strawberry (Rupp et al. 2017), just like B. paeoniae and $B$. tulipae that only or primarily infect peonies (Munoz et al. 2016) and tulips (Coley-Smith and Javed 1972), respectively. The wide host range of $B$. cinerea is presumably due to its evolution of virulence factors that can overcome immunity of many different types of plants (Choquer et al. 2007). As a corollary to this reasoning, strawberry-restricted $B$. fragariae might have fewer effector genes. To evaluate these speculations, we performed comparative genome analyses between $B$. fragariae and other Botrytis species. We identified genes encoding candidate carbohydrate-active enzymes (CAZymes) (Zhang et al. 2018), secreted proteins especially those digestive enzymes required for breaking the plant cell wall, or effectors that are secreted and delivered to host tissues/cells to suppress host immunity from those 12,506 genes of $B$. fragariae, and compared them to those predicted in the genomes of the three other Botrytis species using the same algorithm and criteria. As shown in Table 1, compared with the three host-specific Botrytis species, B. cinerea has a relatively larger predicted CAZyme, secretome, and effectorome. Interestingly, despite having a smaller predicted effectorome relative to that of $B$. cinerea, $B$. fragariae has a significantly higher proportion of species specific effectors (67) $43.5 \%$ ) compared with other Botrytis species (29.0 to $32.9 \%$ ) (Table 1).

The whole genome sequencing project has been deposited to NCBI under accession number JABFCT000000000, and the BioProject and BioSample accession number are PRJNA627915 and SAMN14689424, respectively. The raw Pacbio data and RNA-seq data were deposited to SRA under accession numbers SRR11844330 and SRR11843737.

\section{Literature Cited}

Bendtsen, J. D., Nielsen, H., von Heijne, G., and Brunak, S. 2004. Improved prediction of signal peptides: SignalP 3.0. J. Mol. Biol. 340:783-795.

Choquer, M., Fournier, E., Kunz, C., Levis, C., Pradier, J. M., Simon, A., and Viaud, M. 2007. Botrytis cinerea virulence factors: New insights into a necrotrophic and polyphageous pathogen. FEMS Microbiol. Lett. 277:1-10.

Coley-Smith, J. R., and Javed, Z. U. R. 1972. Germination of sclerotia of Botrytis tulipae, the cause of tulip fire. Ann. Appl. Biol. 71:99-109.

Dean, R., Van Kan, J. A., Pretorius, Z. A., Hammond-Kosack, K. E., Di Pietro, A., Spanu, P. D., Rudd, J. J., Dickman, M., Kahmann, R., Ellis, J., et al. 2012. The top 10 fungal pathogens in molecular plant pathology. Mol. Plant Pathol. 13:414-430.

Dowling, M. E., Hu, M. J., and Schnabel, G. 2018. Fungicide resistance in Botrytis fragariae and species prevalence in the Mid-Atlantic United States. Plant Dis. 102: 964-969.
FAOSTAT. 2018. Production quantity by country. Food and Agriculture Organization of the United Nations Statistics Division. http://www.fao.org/faostat/en/\#data/QC

Holt, C., and Yandell, M. 2011. MAKER2: An annotation pipeline and genome-database management tool for second-generation genome projects. BMC Bioinformatics 12: 491.

Hu, M. J., Cosseboom, S., and Schnabel, G. 2019. atrB-associated fludioxonil resistance in Botrytis fragariae not linked to mutations in transcription factor mrr1. Phytopathology 109:839-846.

Hu, M. J., Cox, K. D., and Schnabel, G. 2016. Resistance to increasing chemical classes of fungicides by virtue of "selection by association" in Botrytis cinerea. Phytopathology 106:1513-1520.

Huang, X., Duan, N., Xu, H., Xie, T. N., Xue, Y. R., and Liu, C. H. 2018. CTAB-PEG DNA extraction from fungi with high contents of polysaccharides. Mol. Biol. 52: 621-628. 
Huerta-Cepas, J., Forslund, K., Coelho, L. P., Szklarczyk, D., Jensen, L. J., von Mering, C., and Bork, P. 2017. Fast genome-wide functional annotation through orthology assignment by eggNOG-Mapper. Mol. Biol. Evol. 34:2115-2122.

Korf, I. 2004. Gene finding in novel genomes. BMC Bioinf. 5:59.

Kretschmer, M., Leroch, M., Mosbach, A., Walker, A. S., Fillinger, S., Mernke, D., Schoonbeek, H. J., Pradier, J. M., Leroux, P., De Waard, M. A., et al. 2009. Fungicide-driven evolution and molecular basis of multidrug resistance in field populations of the grey mould fungus Botrytis cinerea. PLoS Pathog 5:e1000696.

Krogh, A., Larsson, B., von Heijne, G., and Sonnhammer, E. L. 2001. Predicting transmembrane protein topology with a hidden Markov model: Application to complete genomes. J. Mol. Biol. 305:567-580.

Lee, E., Helt, G. A., Reese, J. T., Munoz-Torres, M. C., Childers, C. P., Buels, R. M., Stein, L., Holmes, I. H., Elsik, C. G., and Lewis, S. E. 2013. Web Apollo: A webbased genomic annotation editing platform. Genome Biol. 14:R93.

Munoz, G., Campos, F., Salgado, D., Galdames, R., Gilchrist, L., Chahin, G., and Andrade, O. 2016. Molecular identification of Botrytis cinerea, Botrytis paeoniae and Botrytis pseudocinerea associated with gray mould disease in peonies (Paeonia lactiflora Pall.) in Southern Chile. Rev. Iberoam. Micol. 33:43-47.

Petrasch, S., Knapp, S. J., van Kan, J. A. L., and Blanco-Ulate, B. 2019. Grey mould of strawberry, a devastating disease caused by the ubiquitous necrotrophic fungal pathogen Botrytis cinerea. Mol. Plant Pathol. 20:877-892.

Rhoads, A., and Au, K. F. 2015. PacBio sequencing and its applications. Genom. Proteom. Bioinf. 13:278-289.

Rupp, S., Plesken, C., Rumsey, S., Dowling, M., Schnabel, G., Weber, R. W. S., and Hahn, M. 2017. Botrytis fragariae, a new species causing gray mold on strawberries, shows high frequencies of specific and efflux-based fungicide resistance. Appl. Environ. Microbiol. 83:e00269-17.

Simao, F. A., Waterhouse, R. M., Ioannidis, P., Kriventseva, E. V., and Zdobnov, E. M. 2015. BUSCO: assessing genome assembly and annotation completeness with single-copy orthologs. Bioinformatics 31:3210-3212.
Sperschneider, J., Dodds, P. N., Gardiner, D. M., Singh, K. B., and Taylor, J. M. 2018. Improved prediction of fungal effector proteins from secretomes with EffectorP 2.0. Mol. Plant Pathol. 19:2094-2110.

Stanke, M., Steinkamp, R., Waack, S., and Morgenstern, B. 2004. AUGUSTUS: A web server for gene finding in eukaryotes. Nucleic Acids Res. 32:W309-W312.

Sutton, J. C. 1998. Botrytis fruit rot (gray mold) and blossom blight. Page 28 in: Compendium of Strawberry Diseases. J. L. Maas, ed. American Phytopathological Society, St. Paul, MN.

Ter-Hovhannisyan, V., Lomsadze, A., Chernoff, Y. O., and Borodovsky, M. 2008. Gene prediction in novel fungal genomes using an ab initio algorithm with unsupervised training. Genome Res. 18:1979-1990.

USDA-NASS. 2018. Noncitrus fruits and nuts. National Agricultural Statistics Service. United States Department of Agriculture Economics, Statistics and market information system. https://www.nass.usda.gov/Publications/Ag_Statistics/2017/ index.php.

Valero-Jimenez, C. A., Veloso, J., Staats, M., and van Kan, J. A. L. 2019. Comparative genomics of plant pathogenic Botrytis species with distinct host specificity. BMC Genomics 20:203.

Van Kan, J. A., Stassen, J. H., Mosbach, A., Van Der Lee, T. A., Faino, L., Farmer, A. D., Papasotiriou, D. G., Zhou, S., Seidl, M. F., Cottam, E., et al. 2017. A gapless genome sequence of the fungus Botrytis cinerea. Mol. Plant Pathol. 18:75-89.

Vermeulen, T., Schoonbeek, H., and De Waard, M. A. 2001. The ABC transporter BcatrB from Botrytis cinerea is a determinant of the activity of the phenylpyrrole fungicide fludioxonil. Pest Manag. Sci. 57:393-402.

Xiao, C. L., Chen, Y., Xie, S. Q., Chen, K. N., Wang, Y., Han, Y., Luo, F., and Xie, Z. 2017. MECAT: Fast mapping, error correction, and de novo assembly for singlemolecule sequencing reads. Nat. Methods 14:1072-1074.

Zhang, H., Yohe, T., Huang, L., Entwistle, S., Wu, P., Yang, Z., Busk, P. K., Xu, Y., and Yin, Y. 2018. dbCAN2: A meta server for automated carbohydrate-active enzyme annotation. Nucleic Acids Res. 46:W95-W101. 